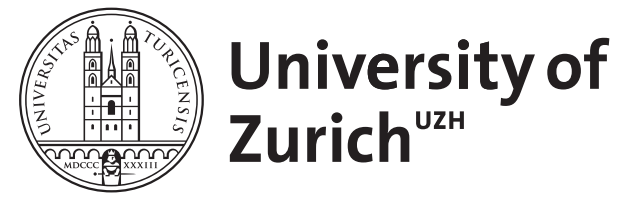
Archive

University of Zurich

University Library

Strickhofstrasse 39

CH-8057 Zurich

www.zora.uzh.ch

Year: 2000

Echtzeit 3D-Ultraschallsystem für transösophageale Herzabbildung und Katheterlokalisation

\author{
Moser, U ; Margadant, F ; Gattiker, F ; Birchler, B ; Niederer, P
}

DOI: https://doi.org/10.1515/bmte.2000.45.s1.27.

Posted at the Zurich Open Repository and Archive, University of Zurich ZORA URL: https://doi.org/10.5167/uzh-155420

Journal Article

Published Version

Originally published at:

Moser, U; Margadant, F; Gattiker, F; Birchler, B; Niederer, P (2000). Echtzeit 3D-Ultraschallsystem für transösophageale Herzabbildung und Katheterlokalisation. Biomedizinische Technik. Biomedical engineering, 45(s1):27-28.

DOI: https://doi.org/10.1515/bmte.2000.45.s1.27. 


\title{
ECHTZEIT 3D-ULTRASCHALLSYSTEM FÜR TRANSÖSOPHAGEALE HERZABBILDUNG UND KATHETERLOKALISATION
}

\author{
${ }^{1,2}$ U. Moser, ${ }^{3}$ F. Margadant, ${ }^{2}$ F. Gattiker, ${ }^{2}$ B. Birchler, ${ }^{1}$ P. Niederer \\ ${ }^{1}$ Institut für Biomedizinische Technik und Med. Informatik, ETH und Universität Zürich, Schweiz \\ ${ }^{2}$ Sulzer Innotec, Sulzer Markets and Technology AG, 8401 Winterthur, Schweiz \\ ${ }^{3}$ Department of Physical Optics, University of Sydney, Sydney NSW 2006, Australia
}

urs.moser@sulzer.com

\section{EINLEITUNG}

Die heutigen Möglichkeiten zur Lokalisation und $\mathrm{Na}-$ vigation von Herzkathetern im schlagenden Herzen sind für die immer anspruchsvolleren minimalinvasiven Verfahren (z.B. HF-Ablation, Injektion von Medikamenten ins Myokard) ungenügend. Von einem verbesserten System muss gefordert werden, dass die Lokalisation in Echtzeit und dreidimensional erfolgen kann. Ausserdem besteht auch der Wunsch nach einem Verfahren, das ohne ionisierende Strahlung auskommt. Diese Bedingungen können am besten von einem speziellen transösophagealen Ultraschall-Navigationssystem erfüllt werden. Es erlaubt, am schlagenden Herzen das Führen und Positionieren von Herzkathetern und anderen wenig-invasiven Instrumenten unter kontinuierlicher 3D Sicht. Dabei findet die Abbildung von Gewebestrukturen und Instrumenten gleichzeitig mit hoher Geschwindigkeit statt, d.h. mit 10 bis 20 3DBildern pro Sekunde. Die Akquisition der UltraschallDaten erfolgt via Ösophagus mit Hilfe eines speziellen Ultraschall-kopfes. Um die Echtzeitfähigkeit des Systems zu gewährleisten, wird im Unterschied zu konventionellen Ultraschallgeräten eine parallele Datenakquisition eingesetzt: Auf 8 parallelen Ebenen wird das interessierende Volumen gleichzeitig abgetastet.

\section{KONZEPT}

Der Ultraschallkopf besteht aus einem rotierenden Zylinder von ca. $12 \mathrm{~mm}$ Durchmesser, auf dem z.B. 24 Ultraschallwandler (Transducer) angebracht sind (Fig. 1). Die Anordnung dieser Transducer ist in 3 Gruppen unterteilt, wobei die einzelnen Transducer einer Gruppe in axialer Richtung auf einer Linie dicht aneinander gereiht sind. Die einzelnen Gruppen sind in Umfangsrichtung um 120 Grad gedreht und in axialer Richtung versetzt angeordnet. Der Versatz beträgt $1 / 3$ des Mittenabstandes zwischen den Transducern innerhalb einer Gruppe. Mit dieser Anordnung können in einem Sektor gleichzeitig 8 Ebenen erfasst werden. Mit einer vollen Umdrehung werden somit 24 Ebenen abgetastet, deren Abstand 1/3 des Transducer-Mittenabstandes beträgt.

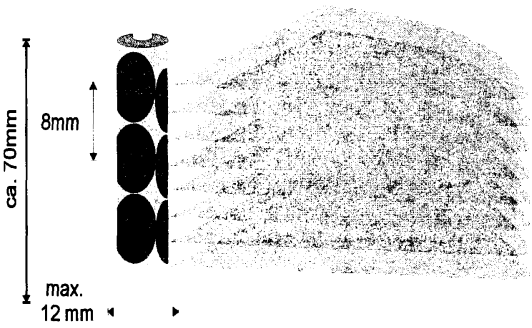

Fig. 1: Transducerkopf mit 3 um $120^{\circ}$ gegeneinander gedrehten linear angeordneten Transducergruppen. Es sind nur 3 statt 8 Transducer pro Gruppe dargestellt. Transducerdurchmesser: $8 \mathrm{~mm}$, Ebenenabstand: $8 / 3 \mathrm{~mm}$

Zur Erzeugung und zum Empfangen der Ultraschallsignale wurde eine spezielle Hardware entwickelt, die die notwendigen Sender enthält und die digitalisierten, gefilterten und dezimierten Empfangssignale via Zwischenpuffer einem leistungs-fähigen Signalprozessor zuführt. Nach geeigneter Aufbereitung werden diese Daten via PCI-Bus in den Systemspeicher des PCs transferiert. Fig. 2 zeigt das Gesamtsystem.

Die Aufgabe des PCs besteht neben der Steuerung des Systems, der Benutzerführung und der Archivierung hauptsächlich in der Visualisierung der akquirierten 3D-Ultraschalldaten. Dazu ist hardwareseitig lediglich eine leistungsfähige Grafikkarte notwendig, die heute für Computerspiele sehr kostengünstig von verschiedenen Herstellern erhältlich ist.

\section{D VISUALISIERUNG IN ECHTZEIT}

Die Visualisierung erfolgt mit mehr als 20 3D - (Stereo-) Bilder pro Sekunde. Sie ist von der Komplexität des Bildes und vom Betrachtungswinkel unabhängig und damit ideal für die geforderte Echtzeitdarstellung. Dies und die hohe Geschwindigkeit wird erreicht, weil das Verfahren auf Texture-Mapping [1] aufbaut, so dass die Fähigkeit heutiger Grafikbeschleuniger, Texturen mit hoher Durchsatzrate und perspektivisch kor- 
rekt darzustellen, ausgenutzen werden kann [2],[3]. Das Verfahren beinhaltet jedoch einen zu [4] zusätzlichen, neuartigen Transformationsschritt, so dass die originalen Zylinderkoordinaten, in denen die Daten aufgezeichnet werden nie verlassen oder umgerechnet werden müssen. Die Umsetzung in die kartesischen Koordinaten des Bildschirms erfolgen erst in der Projektion, nicht jedoch im Volumen. Es werden jeweils stereoskopische Paare der Projektion berechnet. Die Bilder für das linke und das rechte Auge werden zeitsequentiell auf dem Monitor ausgegeben und mittels einer handelsüblichen LCD-Stereobrille ausschliesslich dem jeweiligen Auge zugänglich gemacht. In Zukunft werden auch Monitore zur Verfügung stehen, die ohne Brille eine Stereo-Sicht vermitteln. Der Benutzer erhält mit diesem System einen räumlichen, in Echtzeit animierten Einblick in das schlagende Herz, einschliesslich der Gewebestrukturen und des $\mathrm{zu}$ navigierenden Instrumentes.

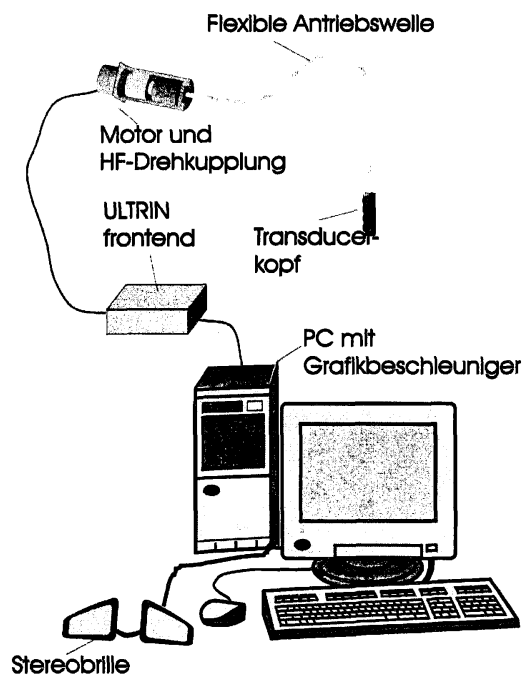

Fig. 2: Das Gesamtsystem in Überblick.

\section{PROJEKTSTATUS}

Es steht eine voll funktionstüchtige Hard- und Software zur Verfügung. Aus Kosten- und Zeitgründen musste jedoch vorläufig auf die in Fig. 2 angedeutete flexible Antriebswelle und den schluckbaren Schallkopf verzichtet werden. Der Prototyp besitzt eine starre Antriebswelle und kann nur im Wasserbad verwendet werden.

\section{ERGEBNISSE}

Es wurden Testmessungen im Wasserbad mit einem Array von $5 \mathrm{MHz}$-Transducern mit $8 \mathrm{~mm}$ Durchmesser durchgeführt. Dabei wurde das Sende-Empfangsmuster in einer senkrecht zur Hauptschallausbreitungsrichtung stehenden Ebene in verschiedenen Entfernungen zum Array untersucht. Erwartungsgemäss verändert sich das Sende-Empfangsmuster eines bestimmten Transducers nicht merklich, wenn mit allen Transducern gleichzeitig anstatt nur mit jenem gesendet wird, der auch als Empfänger dient. Dies bedeutet, dass der parallele Sendebetrieb und die parallele Datenakquisition die Bildqualität nicht merklich beeinträchtigt.

\section{DISKUSSION}

3D Ultraschallgeräte, die durch sequentielles Drehen oder Schwenken der Scanebene ein Volumen abtasten, sind für Navigationsaufgaben am schlagenden Herzen ungeeignet, da die Datenakquisition zu lange dauert [5]. Dagegen erreicht das hier vorgestellte Verfahren mit 8-fach parallelem Abtasten eine Bildfrequenz, die für Echtzeitanwendungen ausreicht. Verglichen mit anderen echtzeitfähigen 3D Ultraschallsystemen, die mit Hilfe von 2D Arrays einen parallelen Empfang erreichen [6], ist unser System wesentlich kostengünstiger, da es mit nur 8 Empfangskanälen auskommt.

\section{LITERATURHINWEISE}

[1] Texture Mapping

The X Journal, March 1995

Kilgard, $\mathrm{M}$.

[2] Direct volume-rendering with shading via threedimensional textures

Van Geldern, A., Wilson, O.

Symposium on Volume Visualization, ACM SIGGRAPH 1996

[3] Accelerating Volume Reconstruction with 3D Texture Hardware

Cullip,U., Neumann,U.

UNC Tech Report TR93-0027, 1993

[4] Fast volume rendering using a shear-warp factorization of the viewing transform

Lacroute,P., Levoy,M.

Computer Graphics

SIGGRAPH Proceedings 1994

[5] Jos R.T.C. Roelandt et. al., "Ultrasonic Dynamic Three-dimensional Visualization of the Heart With a Multiplane Transesohpageal Imaging Transducer", J. of the American Society of Echocardiography, May-June 1994

[6] ECOSCAN, VOLUMETRICS Model 1, http://www.hitachi.echoscan.com/3d/system .html 